\title{
Using Ozone Therapy as an Option for Treatment of COVID-19 Patients: A scoping review
}

\author{
sakineh hajebrahimi ${ }^{1}$, Negar Taleschian-tabrizi ${ }^{1}$, sepideh Karkon-shayan ${ }^{2}$, Fariba \\ pashazadeh $^{1}$, sarvin radvar $^{1}$, ali motamed-sanaye ${ }^{2}$, and Amirreza Tavassoli ${ }^{3}$ \\ ${ }^{1}$ Tabriz University of Medical Sciences \\ ${ }^{2}$ Gonabad University of Medical Sciences \\ ${ }^{3}$ Tabriz University
}

April 27, 2020

\begin{abstract}
Background: Recently, there has been a global demand toward finding new treatments to control the new pandemic of coronavirus (COVID-19). The aim of this systematic review was to study the effects of ozone therapy on COVID-19 patients, as well as reviewing the available supporting evidence. Methods: Electronic databases including MEDLINE (via PubMed), EMBASE, Cochrane Library (CENTRAL), and TRIP, as well as clinical trial registries and preprint sources were searched for published evidence-based articles. In addition, a hand searching was conducted regarding the attained articles published until 6 April 2020 using MeSH and free text keywords with no language limitation. Articles were screened, categorized and extracted for the relative data, which are reported in a descriptive manner. Results: Among 234 articles, 9 were selected to be reviewed for the inclusion criteria. No previously published original articles were found on the efficacy of ozone therapy on COVID-19. Five review studies on the potential role of systemic ozone therapy were found, in which it was concluded that ozone therapy is effective in controlling COVID-19 due to its antiviral, oxygenating, anti-inflammatory, oxidation balancing and immunomodulation effects. Three ongoing clinical trials were registered in China. Moreover, a preliminary report of an ongoing study in Italy on 46 patients (11 intubated and 35 non-intubated) indicated that 39 patients (84\%) showed an improvement for the symptoms of the disease. Conclusion: Despite the promising hypothetical background, opinions of the expert arguing about the benefits of this method, and a preliminary report indicating the effectiveness of ozone therapy as a novel treatment option for COVID-19, there is still not enough evidence to prove it.
\end{abstract}

\section{Hosted file}

Revised Ozone therapy.docx available at https://authorea.com/users/315183/articles/446123-usingozone-therapy-as-an-option-for-treatment-of-covid-19-patients-a-scoping-review 